\title{
An investigation of variable relationships in 3-SAT problems
}

\author{
Olena Kravchuk, Wayne Pullan, John Thornton, and Abdul Sattar \\ School of Information Technology, \\ Griffith University Gold Coast, \\ Qld, 4215, Australia \\ Email: \{o.kravchuk, w.pullan, j.thornton, a.sattar\}@mailbox.gu.edu.au
}

Keywords: Constraints, Search

\begin{abstract}
To date, several types of structure for finite Constraint Satisfaction Problems have been investigated with the goal of either improving the performance of problem solvers or allowing efficient problem solvers to be identified. Our aim is to extend the work in this area by performing a structural analysis in terms of variable connectivity for 3-SAT problems. Initially structure is defined in terms of the compactness of variable connectivity for a problem. Using an easily calculable statistic developed to measure this compactness, a test was then created for identifying 3-SAT problems as either compact, loose or unstructured (or uniform). A problem generator was constructed for generating 3-SAT problems with varying degrees of structure. Using problems from this problem generator and existing problems from SATLIB, we investigated the effects of this type of structure on satisfiability and solvability of 3-SAT problems. For the same problem length, it is demonstrated that satisfiability and solvability are different for structured and uniform problems generated by the problem generator.
\end{abstract}

\section{Introduction}

Non-randomness in Constraint Satisfaction Problems (CSPs) has generated considerable interest in the CSP community [1]. This has resulted in a number of alternative models for generating CSPs with more realistic structures than using random models [2]. However it is still not possible to provide conclusions other than straight-forward ones such as: sometimes structured problems differ from non-structured in their characteristics. Partially such uncertainty is explained by the lack of a clear definition of structure. Although there has been some work on statistically classifying problem structure using symmetry [3], the level of interchangeability [4], clustering coefficient [5] and backbone size ([6], [7]) and approximate entropy [8], generally a problem is called structured if it is not uniformly random. As a consequence, research in the field has produced polar conclusions: in [4] it was noted that real-world structured problems have greater simplicity than randomly generated problems, while in [6] it was suggested that harder problems can be constructed by perturbations of regular problems. In [9] it was demonstrated that structure affects algorithm performance, while in [10] no noticeable effects were reported. It is important to note here that in this previous work, no statistical tests of specific structure have been proposed. Therefore, conclusions on structured 
problems were generally conclusions on the properties of generators, as problems were not tested independently for the structure investigated ([3], [4], [6]).

This study focusses on the k-SAT problem which is a special class of the Conjunctive Normal Form (CNF) problem with precisely $\mathrm{k}$ variables per clause, and has become a popular test-class for the properties of random problems ([11],[12]). Many important characteristics of k-SAT problems such as cross-over points, phase transition regions and satisfiability points have been studied experimentally and analytical [13].

A simple uniform binary model allows one to analyse some phenomena of k-SAT problems. The uniform binary model is a composition of two processes, one which randomly builds or selects clauses and a second which randomly negates the variables [11]. Therefore it is possible to induce a structure, in the sense of non-uniformity, for either the first or the second processes. In this paper we develop a test of non-uniformity in selecting clauses, and investigate the influence of such a structure on the satisfiability and solvability of 3-SAT problems. Satisfiability is the generic property of a problem to have at least one solution, while solvability is solver specific and is the property that the problem is able to be solved by that solver within a certain number of steps.

The remainder of this paper is structured as follows: Section 2 discusses uniform and non-uniform models for random 3-SAT problems and shows that a problem can be considered as a realization of a certain random process. Section 3 introduces a teststatistic for uniformity and investigates the distribution of the statistic for a uniformly generated 3-SAT problem, which is treated as a realization of a non-decreasing, transient discrete Markov chain. We then show examples of applications of the test to groups of problems taken from the SATLIB library [14]. Section 4 presents a structured 3-SAT problem generator using the random process developed in Section 3, introduces parameters into the problem generator and derives testable ranges for these parameters. Section 5 discusses the results obtained for two groups of tests: firstly, a test for differences in satisfiability for uniform and structured problems generated by the problem generator and, secondly, a test on differences in solvability (i.e. effort required to find a solution by a particular solver) for uniform and non-uniform problems. In Section 6 we discuss the effects of structure, in terms of variable connectivity, on the satisfiability and solvability of problems and outline the most important characteristics of the test-statistic and problem generator. Finally some directions for future research are presented.

\section{Uniform and Non-Uniform Binary Models for 3-SAT Problems}

\subsection{A Uniform Binary Model as a Two-Step Model}

A 3-SAT problem is a constraint formula in conjunctive normal form with 3 variables (literals) per clause. We denote the number of clauses in a 3-SAT problem by $M$ and the number of variables by $N$. The uniform binary model (often referred to as the uniform random model [15], or as the exact l-SAT model [10], or as the uniform model [14]) uniformly selects $M$ clauses from N3 possible combinations of variables, and then uniformly negates variables in each selected clause. The uniform binary model can be considered as a realization of two random processes: one governs adjacency of variables and another the negation of variables. By utilising non-uniform rules for how 
to group or negate variables, we can induce structure in either or both of the processes. In this paper we focus only on the induction of structure into the process of grouping of variables (i.e. the selecting of clauses).

\subsection{Definition of a Non-Uniform Binary Model}

In the uniform binary model, all clauses are selected uniformly. That is, there is no underlying reason why any particular group of clauses will be selected in preference to any other group of clauses. Consequently we can define a non-uniform problem as a problem in which there are underlying reasons that cause the condition of uniformity for selecting clauses to be violated. We will categorise non-uniform problems as either loose or compact. A problem is loose if there is a tendency for any two clauses to share at most one variable. A problem is compact if there is a tendency that any two clauses share at least two variables. These definitions of structure can be formalized as follows. Let us denote by $\mathrm{n}\left(c_{i} \cap c_{j}\right)$ the number of common elements in two clauses $c_{i}, c_{j}, \forall i, \forall j, i \neq j$. In a compact problem, the probability $\mathrm{P}(\cdot)$ that any two clauses intersect in more than one variable, is greater than the same probability under the uniform model $\overline{\mathrm{P}}(\cdot)$. In a loose problem, the probability that any two clauses intersect in no less than two variables is greater than the same probability for the uniform binary model. Fig. 1 illustrates both loose and compact problems according to this definition.

$$
\begin{array}{r}
\text { compact: } \mathrm{P}\left(\mathrm{n}\left(c_{i} \cap c_{j}\right)>1\right)>\overline{\mathrm{P}}\left(\mathrm{n}\left(c_{i} \cap c_{j}\right)>1\right), \\
\text { loose: } \mathrm{P}\left(\mathrm{n}\left(c_{i} \cap c_{j}\right)<2\right)<\overline{\mathrm{P}}\left(\mathrm{n}\left(c_{i} \cap c_{j}\right)<2\right) .
\end{array}
$$

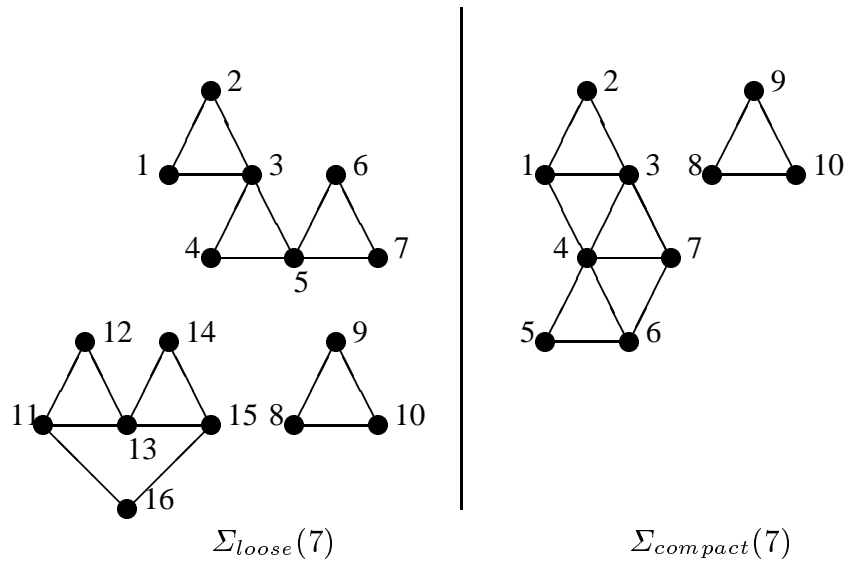

Fig. 1. Planar representation of both loose and compact problems where $M=7$ ( $\Sigma_{\text {loose }}=$ $(\neg 1 \vee 2 \vee 3) \wedge(3 \vee \neg 4 \vee 5) \wedge(5 \vee 6 \vee \neg 7) \wedge(\neg 8 \vee 9 \vee \neg 10) \wedge(11 \vee \neg 12 \vee 13) \wedge$

$\left.(\neg 13 \vee 14 \vee 15) \wedge(\neg 11 \vee \neg 15 \vee \neg 16), N_{0}(7)=21\right)\left(\Sigma_{\text {compact }}=(1 \vee 2 \vee 3) \wedge(\neg 1 \vee 2 \vee\right.$

$\neg 3) \wedge(1 \vee \neg 3 \vee 4) \wedge(3 \vee \neg 7 \vee 4) \wedge(4 \vee 7 \vee \neg 6) \wedge$

$\left.(\neg 4 \vee 5 \vee 6) \wedge(8 \vee 9 \vee \neg 10), N_{0}(7)=14\right)$ 
The definitions in (1) - (2) can be used to develop a non-uniform model. In the nonuniform model, to construct a compact problem containing $M$ clauses, starting from the $i^{t h}$ clause $(i>2, i \ll M)$, in the formula, we will, with high probability, add a clause to the formula if it intersects with at least one already selected clause in no less than two variables. Similarly, for the loose problem of size $M$, starting from the $i^{t h}$ clause, $(i>2, i \ll M)$, we will, with high probability, add a clause to the formula if it intersects with any selected clause in no more than one variable.

In the following section we develop a test on uniformity of 3-SAT problems which detects structure of the type defined by (1) - (2).

\section{Testing for Uniformity}

In Section 2, we have defined non-uniformity in random problems. To detect the structure defined by (1) - (2) requires a test which classifies a problem as either compact, loose or uniform. Therefore, for the test of uniformity, we have the following set of hypotheses:

Null hypothesis: The problem is uniformly randomly generated.

Compact alternative: The problem is not uniform but rather possesses a structure as defined by the compact model (1).

Loose alternative: The problem is not uniform but rather possesses a structure as defined by the loose model (2).

Note that, as for any statistical test, we are looking for some specific type of nonuniformity. If there is another reason for a problem to be non-uniform, our test may not detect this.

\subsection{Zero Step Related Variable Pairs as a Test Statistic}

Definition of Step Related Variable Pairs To carry out the proposed test of uniformity, we require a statistic that is independent of the order of clauses. In this study we have used the number of zero step related variable pairs, $N_{0}(M)$, as the test statistic which is simply the count of the number of pairs of variables that appear at least once in the same clause in the problem. That is, $N_{0}(M)$ is the number of edges, counted without repetitions, which appear in the adjacency graph for the problem. The expected value for $N_{0}(M)$ for a uniform binary 3-SAT problem is given by the recurrence relation shown in Eq. (3) (where $N_{0}(0)=0$ ) and is illustrated by Fig. 2. $N_{0}(M)$ is the first in a family of statistics $N_{k}(M)$ which could be used to classify problems in terms of the step relationships between variables. For example, in Fig. $1, \Sigma_{\text {loose }}(7)$, variables 1 and 2 are zero step related, 2 and 4 are one step related while 2 and 6 are two step related. The general recurrence relation for $N_{k}(M), \forall k \geq 1$ is given by (4) and shown in Fig. 2 for $N_{1}(M), N_{2}(M)$ and $N_{3}(M)$.

$$
E\left[N_{0}(i+1)\right]=N_{0}(i)+3-\frac{3 N_{0}(i)}{N(N-1) / 2} i \geq 0
$$




$$
E\left[N_{k}(i+1)\right]=N_{k}(i)+9 \times 2^{k+1} \frac{i}{N}\left(1-\frac{2}{N(N-1)} \sum_{j=0}^{k} N_{j}(i)\right) \forall k \geq 1, i \geq 0
$$

$N_{0}(M)$ is the most suitable statistic from the $N_{k}(M)$ family to be used as a test statistic
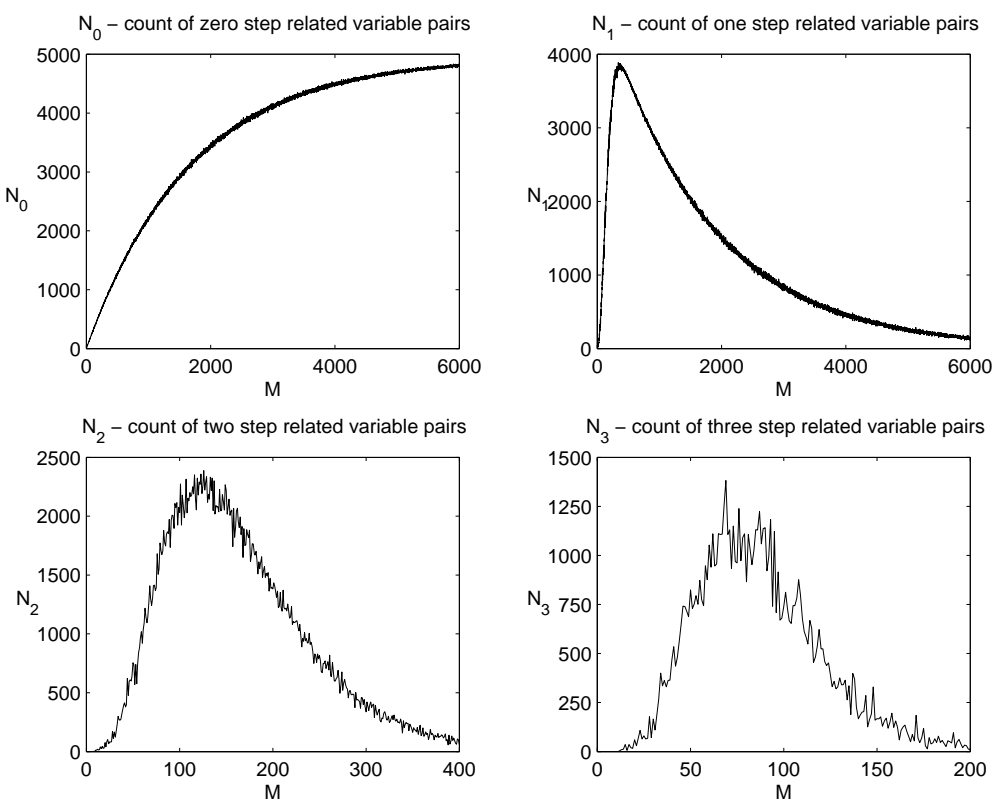

Fig. 2. Counts of step related variable pairs for uniform binary model for $N_{0}(M), N_{1}(M)$, $N_{2}(M)$ and $N_{3}(M)$ where $N=100$

because $N_{2}(M)$ and $N_{3}(M)$ quickly diminish and would require sampling techniques if used for moderate size problems.

For a problem of size $M$, we consider how $N_{0}(M)$ is distributed under the null hypothesis that the problem is uniformly generated. Knowing this null-distribution we can reject or accept the hypothesis of uniformity with a certain level of confidence, measured by the probability to have the given value for $N_{0}(M)$ from the uniform model.

Null-distribution of $N_{0}(M)$ We consider a uniform problem as a realization of a nondecreasing transient discrete Markov chain, so that when uniformly selecting a clause, we can add to the collection of zero step related pairs $k$ ( $k \in\{0,1,2,3\})$ new pairs from $K=\mathrm{N} 2$ pairs. Then the distribution of $N_{0}(M)$ is completely determined by the matrix of transient probabilities on the chain. By the nature of $N_{0}(M)$, the larger the problem, the less the distinction between non-structured and structured problems. In 
the limit, when all pairs of variables are selected, $N_{0}(M)$ converges to $K$, regardless of how the problem was generated. However when $N>10$ and $1 \ll M \ll \mathrm{N} 3$, the transient probability $p_{i k}$ to move from state $i, i \in[3, K]$, to state $i+k$ at the $(n+1)^{t h}$, $n \in[2, M)$, added clause is:

$$
p_{i k}=P\left(N_{0}(n+1)=i+k \mid N_{0}(n)=i\right)=\left(\begin{array}{l}
3 \\
k
\end{array}\right)\left(\frac{i}{K}\right)^{3-k}\left(1-\frac{i}{K}\right)^{k}
$$

For a reasonable problem size, the probability to have at least $i$ zero-related pairs in a problem of size $M, \overline{\mathrm{P}}\left(N_{0}(M) \leq i\right)$ can be calculated by enumeration, and we use this method for calculations in this study.

The cumulative probability of $N_{0}(430)$, shown in Fig. 3, demonstrates the symmetry of the distribution that makes $N_{0}(M)$ appropriate for many statistical tests.

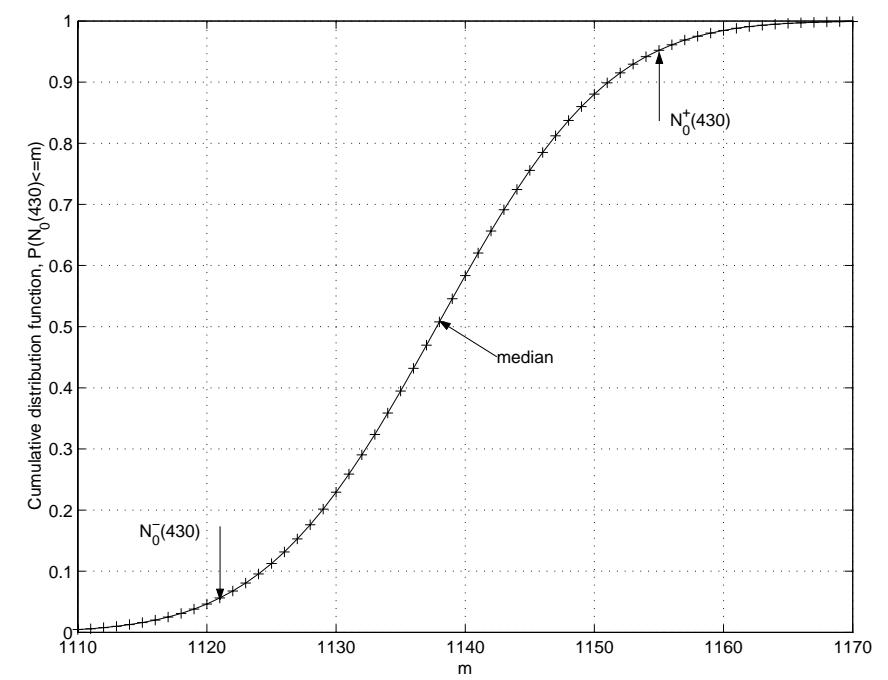

Fig. 3. Cumulative probability of $N_{0}(430)$ where $m$ is zero step related pairs. The median is at 1138 pairs, the critical region is $N_{0}^{-}(430)=1121, N_{0}^{+}(430)=1153$.

For the purpose of our test, we first determine a critical region for $N_{0}(M)$. This is the domain of values of $N_{0}(M)$ where the probability of occurring under the null distribution is less than some value $\alpha$. For our classification of problems as loose and compact, we denote $N_{0}^{+}(M)$ and $N_{0}^{-}(M)$ as the boundaries (loose and compact) of this critical region, and define the boundaries as follows:

$$
\begin{aligned}
& \overline{\mathrm{P}}\left(N_{0}(M) \leq N_{0}^{-}(M)\right) \approx \alpha, \\
& \overline{\mathrm{P}}\left(N_{0}(M)>N_{0}^{+}(M)\right) \approx \alpha,
\end{aligned}
$$

where $\alpha$ is a given confidence level that determines the error we allow for our conclusions. In all experiments reported in this study we use the approximate 5\%-confidence 
level for both alternative hypotheses. The boundaries of the critical region for $M=$ 430 and a confidence level of $5 \%$ are shown in Fig. 3 where $N_{0}^{+}(430)=1153$ and $N_{0}^{-}(430)=1121$.

Uniformity test procedure For a given problem, the test is performed as follows:

1. Determine the number of variables $(N)$ and the number of clauses $(M)$ in the problem;

2. For the pair $(N, M)$, calculate the critical region for the chosen confidence level $\alpha$;

3. Determine the number of zero related pairs $N_{0}(M)$ in the given problem;

4. Reject the hypothesis of uniformity if $N_{0}(M)$ is in the critical region, otherwise, accept the uniformity.

At this point, we have only discussed the test in application to a problem in determining whether the problem is uniformly generated or compact/loose. However, $N_{0}(M)$ could also be utilised for the more general question of problem classification of 3-SAT problems. That is, is a proposed classification of 3-SAT problems justifiable (rather than just a random grouping of problems).

Test of Randomness Procedure As we know the distribution of $N_{0}(M)$, we know the median of the distribution (Fig. 3). Having a group of problems of the same size from the same number of variables, we can test whether the group is a random selection by applying the standard test of randomness [16]. For every problem in the group, the probability to have $N_{0}(M)$ greater or less than the median is equal to 0.5 . Therefore, if in the group we have an unusually large or small number of problems having $N_{0}(M)$ above (or below) the median, we can conclude that the group is not a random selection but has been selected using some criteria related to our definition of problem structure.

\subsection{Tests on problems from SATLIB}

Uniform 3-SAT problems This test was performed using 3-SAT problems taken from SATLIB (Uniform Random 3-SAT, phase transition region, unforced filtered). From the description given in SATLIB of the model generator for this set of problems, we would expect no regularity in the problems so the test should not conclude any evidence that a problem is non-uniformly generated. In other words, if there is any difference in the behaviour of problem solvers, such a difference cannot be explained by problem structure (1) - (2). As shown in Fig. 3, the critical region for uniform problems where $M=430$ and $N=100$ is $N_{0}^{-}(430)=1121, N_{0}^{+}(430)=1155$ that corresponds to the $5 \%$ confidence level. The median of $N_{0}(430)$ is 1138 . Table 1 shows that a sample from the library (uf100-430) is in a very good agreement with our expectations: no structure is demonstrated, and the random sample is indeed random (i.e. there are approximately the same number of problems above and below the median). 
Table 1. $N_{0}(430)$ for a random sample from Uniform Random 3-SAT from SATLIB, the uf100430 series, format uf100-0\#\#\#.cnf; and acceptance (A) or rejection (R) of the null-hypothesis under the critical region: $N_{0}^{-}(430)=1121$ or $N_{0}^{+}(430)=1155$; and the sign test on randomness.

\begin{tabular}{c|ccccccccccccccc}
$\# \# \#$ & 107 & 108 & 119 & 122 & 125 & 132 & 146 & 151 & 170 & 180 & 187 & 216 & 224 & 225 & 228 \\
\hline \hline$N_{0}$ & 1147 & 1139 & 1140 & 1149 & 1147 & 1136 & 1132 & 1133 & 1130 & 1117 & 1139 & 1153 & 1135 & 1139 & 1138 \\
$H_{0}$ & A & A & A & A & A & A & A & A & A & R & A & A & A & A & A \\
Sign & + & + & + & + & + & - & - & - & - & - & + & + & - & + & 0 \\
\hline
\end{tabular}

Random 3-SAT models with controlled backbone size We tested 3-SAT models taken from the SATLIB library with Controlled Backbone Size (CBS) where the backbone of a problem is basically the intersection of all solutions of the problem [7]. It is stated in SATLIB that every CBS problem was uniformly generated. Therefore, similar to what has been discussed for uniform random problems from the SATLIB, we do not expect any detection of structure under the test for any particular CBS problem. However, if we believe that the size of backbone is an informative statistic which is somehow connected to problem structure, we would expect that the group of CBS problems is not a random selection from uniformly generated problems. Table 2 shows that it is unlikely for the group of CBS problems to be a randomly selected group from uniformly generated problems, and therefore, with high confidence $(\alpha<0.5 \%)$, it can be concluded that a backbone size is a valid criteria for the problems' classification.

Table 2. $N_{0}(429)$ for a random sample from problems with controlled backbones for the CBS_k3_n100_m429_b90_\#\#\#.cnf series from SATLIB; acceptance (A) or rejection (R) of the null-hypothesis of uniformity under the critical region: $N_{0}^{-}(429)=1119$ or $N_{0}^{+}(429)=1153$; and the sign test on randomness of the sample from uniformly generated problems (the median of $N_{0}(429)$ is 1136).

\begin{tabular}{c|ccccccccccccccc}
$\# \# \#$ & 107 & 108 & 119 & 122 & 125 & 132 & 146 & 151 & 170 & 180 & 187 & 216 & 224 & 225 & 228 \\
\hline \hline$N_{0}$ & 1123 & 1135 & 1141 & 1130 & 1129 & 1125 & 1123 & 1137 & 1133 & 1132 & 1107 & 1121 & 1124 & 1120 & 1125 \\
$H_{0}$ & A & A & A & A & A & A & A & A & A & A & R & A & A & A & A \\
Sign & - & - & + & - & - & - & - & + & - & - & - & - & - & - & - \\
\hline
\end{tabular}

\section{Problem Generator with Prescribed Non-Uniformity}

\subsection{Markov Chain Underlying the Problem Generator}

The uniform model can be considered as a realization of a random process of adding pairs into zero step relationships for every new clause selected. The process is a nondecreasing discrete Markov chain with finitely many states. Starting from some group of selected clauses, the chain can be considered as homogeneous, with transition probabilities given by (5). 


\subsection{Problem Generator}

Problem Generator Algorithm The problem generator performs the following steps to generate a problem of $M$ clauses from $N$ variables:

1. Uniformly selects a clause from all possible clauses.

2. Uniformly negates variables in the clause.

3. If the clause has not already been added, with some probability $p$ (see below) add the clause.

4. Repeats Steps 1 - 4 until $M$ clauses have been selected.

Problem Generator Parameters The problem generator is a random generator with six parameters $G(N, M, \Pi)$, where $\mathrm{N}$ is the number of variables, $\mathrm{M}$ is the number of clauses, and $\Pi=\left(p_{0}, p_{1}, p_{2}, p_{3}\right)$ are probabilities to add a clause if it, correspondingly, adds $0,1,2,3$ new zero step related pairs $(G(N, M,(1,1,1,1))$ generates a uniform binary problem). By using appropriate probabilities $p_{i}, i \in[0,3]$, the problem generator will either produce a loose or compact problem. The generated problem is then evaluated using the test discussed in Section 3 to check that the problem is indeed structured. From a sensitivity analysis performed on the generator, we have determined that when $p_{i} \in(0.0-0.5), i \in[0,3]$, the generated problems are structured.

\section{Tests on Properties of Uniform and Non-Uniform 3-SAT Problems}

In this section we analyze satisfiability and solvability of structured and uniform problems. For two classes of problems, we say that class A is more satisfiable than class B if the probability for a randomly selected problem in class A to be satisfiable is greater than the same probability for a randomly selected problem in class B. Similarly, we say that class A has higher solvability under problem solver C than class B if the probability for a randomly selected problem from class A to be solved in a fixed number of steps by problem solver $\mathrm{C}$ is higher than the same probability for a randomly selected problem from class B.

\subsection{Test on Similarity of Satisfiability of Uniform and Non-Uniform Problems from the Generator}

We aim to test whether the structure as defined in (1) - (2) affects the satisfiability of problems. For two samples $A$ and $B$, we will test the hypothesis that there is no difference in satisfiability of problems from $A$ and $B$ against the hypothesis that the probability that an arbitrary unsatisfiable problem from the joined sample, $A+B$, belongs to $A$ does not equal $1 / 2$. We will apply the one-sided U-test with tied ranks [16], on experimental data gathered in Table 3. This test allows one to reject the hypothesis if sample $A$ s distribution is shifted either left or right with respect to sample $B$ s distribution. That is, we reject the hypothesis of similarity because sample $A$ is from more (less) satisfiable class than sample $B$. 
In other studies, for example [15], it has been noted that problem satisfiability changes in the so-called transition region $(M / N \approx 4.3)$. To avoid this effect, our experiments are for $M=400$ and $N=100$. Five groups of problems were generated using the parameters defined in Section 4 and all problems were tested for uniformity. The problems were uniform $G(100,400,(1,1,1,1))$, compact $G(100,400,(0,1,1,0.1))$ and loose $G(100,400,(0,0,1,1))$ for which no trivial insolvability was allowed. To demonstrate that the difference, if any, is not because of $p_{0}=0$, additional compact $G(100,400,(1,1,0,0.1))$ and loose $G(100,400,(1,1,0,1))$ groups of problems were used. The test was performed on random samples from the generated groups. Table 3 shows the counts of unsatisfiable problems, in groups of 10 problems generated with different parameters of the generator. In Table 3, problems are classified loose, compact and uniform for the critical region $N_{0}^{-}(400)=1052, N_{0}^{+}(400)=1084$. With

Table 3. Number of unsatisfiable problems in series of 20 random samples in 10 problems of 400 clauses from 100 variables for different degrees of structure $\left(N_{0}^{+}(400)=1084, N_{0}^{-}(400)=\right.$ 1052); and the rejection of the hypothesis of similarity because of lower (L) satisfiability.

\begin{tabular}{|c|c|c|c|c|c|}
\hline $\bar{\Pi}$ & No unsatisfiable problems & $\min N_{0}$ & $\max N_{0}$ & class & te \\
\hline $1-1-1-1$ & $\begin{array}{|lllllllllllllllllllll|} & 1 & 0 & 1 & 2 & 3 & 0 & 1 & 0 & 1 & 0 & 0 & 0 & 0 & 0 & 0 & 1 & 1 & 4 & 0\end{array}$ & 1052 & 1081 & uniform & \\
\hline $0-1-1-0.1$ & 4674446543764559 & 868 & 904 & pact & \\
\hline $0-0-1-1$ & 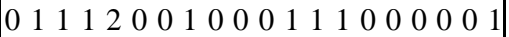 & 1084 & 1100 & loose & \\
\hline $1-1-0-0.1$ & 12211233423411313042 & 914 & 951 & compact & \\
\hline $1-1-0-1$ & {$\left[\begin{array}{llllllllllllllllllll}0 & 0 & 0 & 1 & 1 & 0 & 0 & 0 & 1 & 0 & 2 & 0 & 0 & 0 & 0 & 0 & 0 & 0 & 1 & 1\end{array}\right]$} & 1120 & 1161 & loose & \\
\hline
\end{tabular}

$\alpha=5 \%$, the test cannot reject the hypothesis of similarity between uniform and loose problems. However, we would not expect unsatisfiability for problems of 400 clauses from both the classes. At this size, it is more interesting to compare the compact class with loose and uniform as well. With $\alpha=5 \%$, the test rejected the hypothesis on similarity between compact and uniform problems and between compact and loose problems. Therefore, we can say that our experimental data confirms that, for the same problem size, loose and uniform problems have greater satisfiability than compact problems.

\subsection{Test on Similarity in Solvability for Uniform and Non-Uniform Problems}

Our goal is to determine if problem structure, as defined by (1) - (2), affects problem solvability. For two samples $A$ and $B$ from two classes of problems, we test the hypothesis that there is no difference in solvability of these problem classes against the hypothesis that either of the problem classes is more difficult, i.e. the probability that a problem from sample $A$ is harder than an arbitrary problem from sample $B$ does not equal 0.5. By applying the one-sided U-test, [16], on experimental data gathered in Table 4 we will be able to state that the hypothesis of similarity can be rejected because sample $A$ is from a more difficult (easier) class than sample $B$.

For the reasons given in the test on similarity in satisfiability, we tested this hypotheses on five groups of problems: $G(100,400,(1,1,1,1)), G(100,400,(0,1,1,0.1))$, 
$G(100,400,(0,0,1,1)), G(100,400,(1,1,0,0.1))$, and $G(100,400,(1,1,0,1))$, testing uniform problems against compact and loose problems, and compact problems against loose problems.

Table 4 shows the average number of flips performed by MaxAge, a local search algorithm introduced in [17], for the five groups of problems. Even at $10 \%$ confidence, the test cannot reject the hypothesis of similarity between uniform and loose problems. However, the test concludes, with $5 \%$-confidence, that there is a difference between compact and uniform and also between compact and loose problems. We can say that our experimental data rejects the hypothesis of similarity in solvability between compact and loose or uniform problems because compact problems are more difficult to solve.

Table 4. Average number of flips for MaxAge [17] in random samples of 15 problems of 400 clauses from 100 variables for different degrees of structure $\left(N_{0}^{+}(400)=1084, N_{0}^{-}(400)=\right.$ $1052)$; and the rejection of the hypothesis of similarity because of lower (L) solvability.

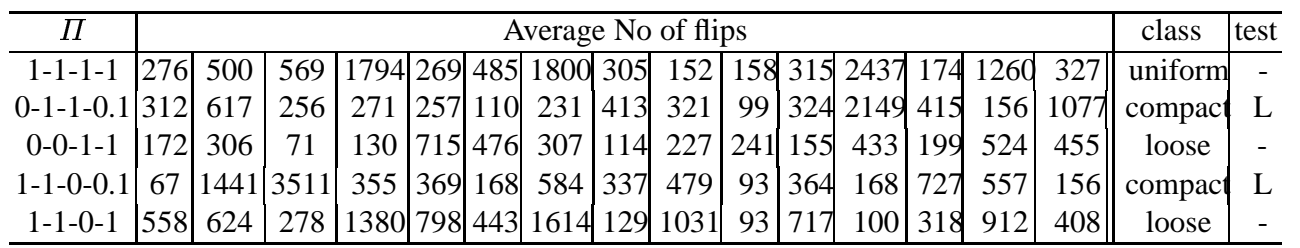

\section{Discussion and Conclusions}

In this paper we have started to address the question of the effects of structure on the properties of SAT problems. A classification of k-SAT problems as either uniform or compact or loose on the basis of variables connectivity has been introduced and justified. This classification method has been developed as a test on problem uniformity based on a simple statistic, the count of the number of zero step related variable pairs. With this test, for problems of moderate size, we have shown that compact problems have low satisfiability and they are more difficult to solve than uniform or loose problems for a local search algorithm. Also, there is no significant difference in solvability and satisfiability between loose and uniform problems. It has been discussed that the same statistic may be used to justify different classifiers. In particular, we have shown that, in terms of variable connectivity, the size of a backbone is a proper classifier for SAT problems.

Non-uniformity in a SAT problem can be induced either through connections between variables or through restrictions on values in the variable domain. We have introduced possible candidates for classifying structures that are of a higher order than the one used in this paper and feel that more sensitive structural analysis could be built 
using such classifiers in a manner similar to that used in this paper. Addressing structure within the domain of variables would also seem to be a logical extension for the structural analysis of SAT problems.

Another goal is to be able to identify problem structure that consists of a combination of both loose and compact substructures. Such structure should allow the application of more generic problem solvers, such as Genetic Algorithms, which should be able to identify and utilise substructures within a SAT problem.

\section{References}

1. Gent I.P., MacIntyre E., Prosser P., Smith B.M., and Walsh T. Random Constraints Satisfaction: flaws and structure. Constraints 2001, Vol.6: 345 - 372.

2. Walsh T. Search on High Degree Graphs. Proceedings of the 17th International Joint Conference on Artificial Intelligence (IJCAI'01) 2001; 266 - 274.

3. Aloul F.A., Ramani A., Markov I.L., Sakallah K.A. Solving difficult SAT instances in the presence of symmetry.Proceedings of Design Automation Conference, University of Michigan, 2002.

4. Beckwith A.B., Choueiry B.Y., H. Zou. How the level of interchangeability embedded in a finite constraint satisfaction problem affects the perfomance of search. Proceedings of the 14th Australian Joint Conference on Artificial Intelligence (AI 2001) 2001; 50 - 61.

5. Walsh T. Search in a small world. Proceedings of the 16th International Joint Conference on Artificial Intelligence 1999; 1172 - 1176.

6. Kautz H., Ruan Y., Achlioptas D., Gomes C., Selman B., Stickel M. Balance and filtering in structured satisfiable problems. Proceedings of the 17th International Joint Conference on Artificial Intelligence 2001; 351 - 358.

7. Parkles A.J. Clustering at the phase transition. Proceedings of the 14th National Conference on Artificial Intelligence 1997; 340-345.

8. Hogg T. Which Search Problems Are Random? Proceedings of AAAI98; 1998; 438-443.

9. Gomes C.P., Selman B. Problem structure in the presence of perturbations. Proceedings of AAAI/IAAI 1997; 221-226.

10. Gu J. Global optimization for Satisfiability (SAT) problem. IEEE Trans. on Data and Knowledge Engineering 1994; Vol.6: 361 - 381.

11. Achlioptas D., Molloy M.S.O., Kirousis L.M., Stamatiou Y.C., Kranakis E., Krizanc D. Random Constraint Satisfaction: a more accurate picture. Constraints 2001; Vol.6: 329 - 344.

12. Cook S.A., Mitchell D.G. Finding hard instances of the satisfiability problem: a survey. In: $\mathrm{Du}, \mathrm{Gu}$, Pardalos (eds.): Satisfiability Problems: Theory and Applications, Vol.5. Americam Mathematical Society, 1997; 1- 17.

13. S.Kirkpatrick, G. Gyorgyi, N. Tishby, and L. Troyansky, The Statistical Mechanics of ksatisfaction, Proceeedings of Advances in Neural Information Processing Systems 1994; 439 $-446$.

14. SATLIB - satisfiability library, main site, http://www.satlib.org

15. Crawford J.M., Auton L.D. Experimental results on the crossover point in satisfiability problems. Proceedings of the 11th National Conference on Artificial Intelligence 1993; 21 - 27.

16. Sachs L. Applied Statistics: a Handbook of Techniques, 2nd Edition, Springer-Verlag, NY, 1984.

17. Thornton J., Pullan W., Terry J. Towards fewer parameters for SAT clause weighting algorithms. Research Report INT-02-02, Griffith University, 2002. 